\title{
Dose invasive apocrine adenocarcinoma has worse prognosis than invasive ductal carcinoma of breast: evidence from SEER database
}

\author{
Ning Zhang ${ }^{1}$, Hanwen Zhang ${ }^{1}$, Tong Chen ${ }^{1}$, Qifeng Yang ${ }^{1,2}$ \\ ${ }^{1}$ Department of Breast Surgery, Qilu Hospital, Shandong University, Jinan, 250012, Shandong Province, People's Republic \\ of China \\ ${ }^{2}$ Pathology Tissue Bank, Qilu Hospital, Shandong University, Jinan, 250012, Shandong Province, People's Republic of China \\ Correspondence to: Qifeng Yang, email: qifengy_sdu@163.com \\ Keywords: apocrine adenocarcinoma, breast cancer, surveillance, epidemiology and end result (SEER), prognosis \\ Received: January 09, $2017 \quad$ Accepted: February 13, $2017 \quad$ Published: February 21, 2017 \\ Copyright: Zhang et al. This is an open-access article distributed under the terms of the Creative Commons Attribution License (CC-BY), \\ which permits unrestricted use, distribution, and reproduction in any medium, provided the original author and source are credited.
}

\section{ABSTRACT}

Background: Invasive apocrine adenocarcinoma (AAC) of breast is a rare histopathological subtype of breast carcinomas. We aim to investigate the different characteristics and prognostic outcomes between AAC and invasive ductal carcinoma (IDC) of breast cancer.

Results: AAC patients presented with older ages, more aggressive behaviors, lower ER and PR proportions, higher HER2 amplification rates and less application of breastconserving therapy and adjuvant chemotherapy compared to IDC patients. Long-term OS and DSS were both worse in ACC patients $(p=0.006, p=0.012$ respectively) than in IDC patients by Kaplan-Meier analysis. However, no significant difference was detected in DSS $(p=0.181)$ and OS $(p=0.116)$ between the matched two histological subtypes. Further subgroup analysis indicated that AJCC stage, ER status, PR status and HER2 status may be principal confounders for AAC prognosis.

Materials and Methods: With accession to the Surveillance, Epidemiology and End Result (SEER) database, a total of 260,596 patients met the eligibility criteria. Clinicopathological characteristics were compared between groups using Chi-square test. Univariate and multivariate analyses were applied to evaluate the overall survival (OS) and disease-specific survival (DSS). Subgroup analyses summarized the hazard ratio (HR) of AAC versus IDC using a forest plot.

Conclusions: AAC had unique clinicopathological characteristics and it tended to be a more aggressive type than IDC. However, the worse prognosis was diminished after matching for demographic and clinicopathological factors. Deeper insights into AAC are in need to contribute to individualized and tailored therapy, which thereby may improve clinical management and outcomes.

\section{INTRODUCTION}

Invasive apocrine adenocarcinoma (AAC) of breast is a rare histopathologically defined subtype of breast carcinoma, which is morphologically characterized by abundant eosinophilic and granular cytoplasm, large nuclei with prominent nucleoli, and distinctive cell membrane by hematoxylin and eosin (H\&E) staining [1]. Furthermore, they tend to exhibit a characteristic hormone receptor profile: estrogen receptor (ER)-negative, progesterone receptor (PR)-negative and androgen receptor (AR)positive, either human epidermal growth factor receptor-2
(HER2)-positive or (epidermal growth factor receptor) EGFR-positive [1-3]. Although the immunohistochemical characteristics are helpful for the proper recognition of the apocrine carcinomas, the presence of malignant apocrine cells in more than $90 \%$ of the tumor population strictly defines AAC of breast [4].

According to reported data, invasive ductal carcinoma (IDC, non-specific type, NST) and invasive lobular carcinoma account for about $75 \%$ and $15 \%$ of all invasive breast carcinoma, respectively [5]. Nevertheless, AAC constitutes between 0.3 and $4 \%$ of invasive breast carcinoma [4, 6-8]. Due to rarity of this entity of breast 
cancer, clinicopathological characteristics and prognoses of patients with AAC were only reported in limited number of studies: either case reports or studies recruiting a small number of patients. Consequently, the available data are sometimes contradictory. In addition, the prognostic values of demographic and clinicopathological characteristics in AAC therefore remain unclear. Matsuo et al. reported patients with AAC were older than those with IDC [9]. However, they only involved 12 patients in their study. Tanaka et al. found lower frequency of axillary nodal involvement in AAC compared to IDC [10]; while Dreyer et al. showed 7 out of 14 apocrine breast carcinomas with positive lymph node status [11]. With respect to prognosis, some of these studies indicated better prognosis in AAC than IDC patients [12-15]; while in some other studies, no significant difference or even poor survival outcome of AAC was detected compared to non-AAC [7, 10, 16-20]. Considering the absence of comprehensive understanding of AAC, AAC managements are currently based on evidence from studies of IDC, which sometimes may be inappropriate. Identifying the prognostic factors of AAC would help to acquire a better knowledge of the disease and make better therapeutic guidelines. Therefore, it is of great importance to clarify the clinicopathological characteristics and prognostic factors of AAC in a large population.

To gain better knowledge of clinicopathological characteristics and prognostic differences between AAC and IDC, we conducted the present study utilizing the Surveillance, Epidemiology, and End Results (SEER) database. In all, 840 patients with AAC were collected in our study, which contained the largest number of AAC patients compared to other published studies to our best knowledge. We aimed at determining the prognostic factors that may account for survival differences between these two histological subtypes of breast cancer.

\section{RESULTS}

\section{Demographics and clinical characteristics of study population}

A total of 260,596 patients met the eligibility criteria for our study, including $840(0.32 \%)$ AAC patients and $259,756(99.68 \%)$ IDC patients. The demographics, tumor and treatment type characteristics were summarized and compared between the two cohorts in Table 1. Significant differences were found in demographics including age and race, tumor characteristics including grade, tumor size, LN status, AJCC stage, ER status, PR status and HER2 status and treatment including surgery type and radiation by comparing the two histological subtypes. AAC patients showed an older age at diagnosis (50-79 years, 81.1\% vs. $70.9 \%$, respectively; $p<0.001$ ) and tended to have a significantly lower proportion of white race $(75.6 \%$ vs. $79.1 \%$, respectively; $p=0.013$ ) than IDC patients.
AAC patients presented more frequently with larger tumors (tumor size $>5 \mathrm{~cm}, 7.4 \%$ vs. $5.0 \%$, respectively; $p<0.001)$ and more grade II and III+UD tumors, namely poorly differentiated tumors (grade II: $46.4 \%$ vs. $39.3 \%$; grade III+UD: $43.2 \%$ vs. $39.3 \%$, respectively; $p<0.001)$. In addition, the rate of positive LN in AAC patients is higher than that in IDC patients $(37.4 \%$ vs. $32.3 \%$, respectively; $p=0.001)$. Collectively, it comes naturally that AJCC stage III patients account for higher proportion in AAC patients than in IDC patients $(17.6 \%$ vs. $12.3 \%$, respectively; $p<0.001)$. A larger proportion of AAC patients were detected with negative ER status (70.1\% vs. $22.8 \%$, respectively; $p<0.001)$ and negative PR status (76.8\% vs. $32.9 \%$, respectively; $p<0.001)$ than IDC patients. However, morepositive HER2 (7.6 vs. $6.8 \%$, respectively; $p<0.001$ ) status was shown in AAC patients than in IDC patients. Treatment also diverged between both groups. Breast conservation surgery (BCS) and adjuvant radiotherapy were less often applied on AAC patients than IDC patients $(55.0 \%$ vs. $60.1 \%$, respectively; $p=0.011$. $52.4 \%$ vs. $56.9 \%$, respectively; $p=0.026$ ).

\section{Comparison of survival between IDC and AAC patients}

Kaplan-Meier plots were used to evaluate overall survival (OS) and disease-specific survival (DSS) in these two histological subtypes (Figure 1). As the plots illustrated, OS and DSS were both worse in ACC patients ( $p=0.006, p=0.012$ respectively) than in IDC patients. In order to further investigate the effects of prognostic factors of OS and DSS, a multivariate analysis by Cox proportional hazards model was performed (Table 2). For both DSS and OS, the multivariate analysis validated that older age at diagnosis, black race, not married status, grade II /III and UD, tumor size $>2 \mathrm{~cm}$, positive lymph node status were associated with poor outcomes, while ER positivity and PR positivity, BCS and radiation were protective factors for DSS. However, AAC histology was found not to be an independent prognostic factor after multivariate analysis in Cox proportional hazard model (AAC vs. IDC, $\mathrm{HR}=0.834,95 \% \mathrm{CI}=0.695-1.002$, $p=0.052(\mathrm{OS}) ; \mathrm{HR}=0.800,95 \% \mathrm{CI}=0.639-1.001$, $p=0.051$ (DSS); respectively).

\section{Survival analysis in matched groups}

To ensure that baseline differences in demographics and clinical characteristics across the two histological subtypes do not account for the outcome discrepancies, we carried out a 1:1 (IDC/AAC) matched case-control analysis using the propensity score matching method. A group of 1,680 patients were obtained, including 840 patients for each histological type (Table 3 ). In the matched groups, only tumor grade $(p<0.001)$ and tumor size $(p=0.034)$ were significantly different between AAC patients and IDC patients. Furthermore, no significant difference 
Table 1: Characteristics of patients from the SEER database by histologic subtype, AAC vs IDC

\begin{tabular}{|c|c|c|c|c|}
\hline & $\begin{array}{c}\text { AAC, } n=840 \\
(\%)\end{array}$ & $\begin{array}{c}\text { IDC, } n=259,756 \\
(\%)\end{array}$ & $\begin{array}{c}\text { Total, } n=260,596 \\
(\%)\end{array}$ & $P$-Value ${ }^{a}$ \\
\hline $\begin{array}{l}\text { Median follow-up } \\
\text { (months) (IQR) }\end{array}$ & $61(31.25-94)$ & $50(23-83)$ & $50(23-83)$ & \\
\hline \multicolumn{5}{|l|}{ Age at diagnosis (years) } \\
\hline $18-49$ & $159(18.9)$ & $75,714(29.1)$ & $75,873(29.1)$ & \multirow[t]{2}{*}{$<0.001$} \\
\hline $50-79$ & $681(81.1)$ & $184,042(70.9)$ & $184,723(70.9)$ & \\
\hline \multicolumn{5}{|l|}{ Race } \\
\hline White & $635(75.6)$ & $205,447(79.1)$ & $206,082(79.1)$ & \multirow[t]{4}{*}{0.013} \\
\hline Black & $99(11.8)$ & $28,295(10.9)$ & 28,394 (10.9) & \\
\hline Others $^{\mathrm{b}}$ & $104(12.4)$ & $24,625(9.5)$ & $24,729(9.5)$ & \\
\hline Unknown & $2(0.2)$ & $1,389(0.5)$ & $1,391(0.5)$ & \\
\hline \multicolumn{5}{|l|}{ Marital status } \\
\hline Married & $496(59.0)$ & $156,646(60.3)$ & $157,142(60.3)$ & \multirow[t]{3}{*}{0.131} \\
\hline Not married ${ }^{c}$ & $321(38.2)$ & $93,185(35.9)$ & $93,506(35.9)$ & \\
\hline Unknown & $23(2.7)$ & $9,925(3.8)$ & $9,948(3.8)$ & \\
\hline \multicolumn{5}{|l|}{ Laterality } \\
\hline Left & $434(51.7)$ & $131,609(50.7)$ & $132,043(50.7)$ & \multirow[t]{3}{*}{0.810} \\
\hline Right & $406(48.3)$ & $128,119(49.3)$ & $128,525(49.3)$ & \\
\hline Only one side, NOS & $0(0.0)$ & $28(0.0)$ & $28(0.0)$ & \\
\hline \multicolumn{5}{|l|}{ Grade } \\
\hline I & $59(7.0)$ & 48,702 (18.7) & 48,761 (18.7) & \multirow[t]{4}{*}{$<0.001$} \\
\hline II & $390(46.4)$ & $102,176(39.3)$ & $102,566(39.3)$ & \\
\hline $\mathrm{III}+\mathrm{UD}^{\mathrm{d}}$ & $363(43.2)$ & $102,015(39.3)$ & $102,378(39.3)$ & \\
\hline Unknown & $28(3.3)$ & $6,863(2.6)$ & $6,891(2.6)$ & \\
\hline \multicolumn{5}{|l|}{ Tumor size $(\mathrm{cm})$} \\
\hline$\leq 2$ & $483(57.5)$ & $165,658(63.8)$ & $166,141(63.8)$ & \multirow[t]{4}{*}{$<0.001$} \\
\hline$>2$ and $\leq 5$ & $285(33.9)$ & $79,527(30.6)$ & $79,812(30.6)$ & \\
\hline$>5$ & $62(7.4)$ & $13,053(5.0)$ & $13,115(5.0)$ & \\
\hline Unknown & $10(1.2)$ & $1,518(0.6)$ & $1,528(0.6)$ & \\
\hline \multicolumn{5}{|l|}{ LN status } \\
\hline Negative & 497 (59.2) & $169,043(65.1)$ & $169,540(65.1)$ & \multirow[t]{3}{*}{0.001} \\
\hline Positive & $314(37.4)$ & $83,935(32.3)$ & $84,249(32.3)$ & \\
\hline Unknown & $29(3.5)$ & $6,778(2.6)$ & $6,807(2.6)$ & \\
\hline \multicolumn{5}{|l|}{ AJCC stage } \\
\hline I & $380(45.2)$ & $130,690(50.3)$ & $131,070(50.3)$ & \multirow[t]{3}{*}{$<0.001$} \\
\hline II & $312(37.1)$ & $97,082(37.4)$ & $97,394(37.4)$ & \\
\hline III & $148(17.6)$ & $31,984(12.3)$ & $32,132(12.3)$ & \\
\hline \multicolumn{5}{|l|}{ ER status } \\
\hline Negative & $589(70.1)$ & $59,323(22.8)$ & $59,912(23.0)$ & \multirow[t]{2}{*}{$<0.001$} \\
\hline Positive & $251(29.9)$ & $200,433(77.2)$ & $200,684(77.0)$ & \\
\hline
\end{tabular}




\begin{tabular}{|c|c|c|c|c|}
\hline \multicolumn{5}{|l|}{ PR status } \\
\hline Negative & $645(76.8)$ & $85,524(32.9)$ & $86,169(33.1)$ & \multirow[t]{2}{*}{$<0.001$} \\
\hline Positive & $195(23.2)$ & $174,232(67.1)$ & $174,427(66.9)$ & \\
\hline \multicolumn{5}{|l|}{ HER2 status } \\
\hline Negative & $187(22.3)$ & $86,291(33.2)$ & 86,478 (33.2) & \multirow[t]{4}{*}{$<0.001$} \\
\hline Positive & $64(7.6)$ & $17,740(6.8)$ & $17,804(6.8)$ & \\
\hline Borderline & $7(0.8)$ & 2,362 (0.9) & 2,369 (0.9) & \\
\hline Unknown & $582(69.3)$ & $153,363(59.1)$ & $153,945(59.1)$ & \\
\hline \multicolumn{5}{|l|}{ Surgery type } \\
\hline Mastectomy & $377(44.9)$ & $103,403(39.8)$ & $103,780(39.8)$ & \multirow[t]{3}{*}{0.011} \\
\hline BCS & $462(55.0)$ & $156,053(60.1)$ & $156,515(60.1)$ & \\
\hline Unknown & $1(0.1)$ & $300(0.1)$ & $301(0.1)$ & \\
\hline \multicolumn{5}{|l|}{ Radiation } \\
\hline No & $365(43.5)$ & $103,177(39.7)$ & $103,542(39.7)$ & \multirow[t]{3}{*}{0.026} \\
\hline Yes & $440(52.4)$ & $147,735(56.9)$ & $148,175(56.9)$ & \\
\hline Unknown & $35(4.2)$ & $8,844(3.4)$ & $8,879(3.4)$ & \\
\hline
\end{tabular}

$\mathrm{AJCC}=$ American Joint Committee on Cancer, $\mathrm{BCS}=$ breast conserving surgery, ER=estrogen receptor, $\mathrm{PR}=$ progesterone receptor, HER2 = human epidermal growth factor receptor 2, IDC = infiltrating ductal carcinoma, IQR=interquartile range, $\mathrm{LN}=$ lymph node, $\mathrm{NOS}=$ no other specific, $\mathrm{UD}=$ undifferentiated, SEER = Surveillance, Epidemiology and End Results. ${ }^{a} P$-value of the Chi-square test comparing the AAC and IDC groups.

${ }^{b}$ Including American Indian/Alaskan native, and Asian/Pacific Islander, and others-unspecified.

'Including divorced, separated, single (never married), unmarried, domestic partner and widowed.

Including grade 3 and undifferentiated.

was detected in OS $(p=0.116)$ and DSS $(p=0.181)$ between the two histological subtypes (Figure 2).

\section{Subgroup analysis}

In subgroup analysis of both OS and DSS, prognosis for patients with older age at diagnosis, white race and married status were worse in AAC histological type than in

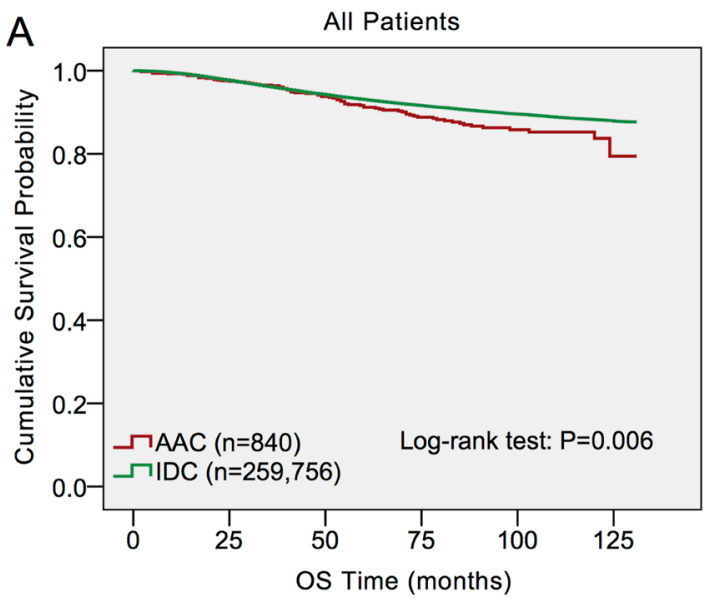

IDC type (AAC vs. IDC, for older age, $\mathrm{HR}=1.252,95 \%$ $\mathrm{CI}=1.028-1.525(\mathrm{OS}) ; \mathrm{HR}=1.347,95 \% \mathrm{CI}=1.045-1.736$ (DSS); for white race, $\mathrm{HR}=1.332,95 \% \mathrm{CI}=1.078-1.646$ (OS); $\mathrm{HR}=1.392,95 \% \mathrm{CI}=1.071-1.810(\mathrm{DSS})$; for married status, $\mathrm{HR}=1.363,95 \% \mathrm{CI}=1.060-1.752(\mathrm{OS})$; $\mathrm{HR}=1.388,95 \% \mathrm{CI}=1.028-1.872(\mathrm{DSS}))($ Table 4$)$. Similarly, in subgroup of well differentiated diseases, AAC patients also showed poor prognosis than IDC patients

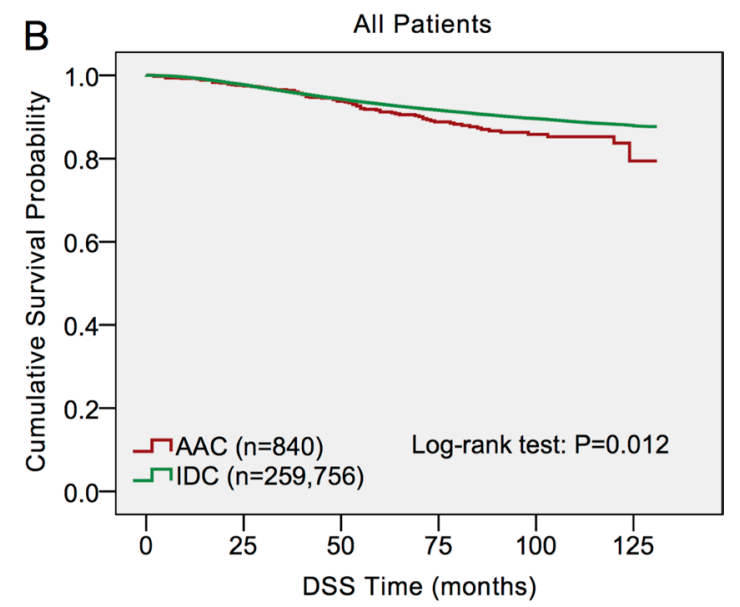

Figure 1: Log-rank test for breast cancer overall survival (OS) and disease-specific survival (DSS) to compare invasive apocrine adenocarcinoma (AAC) to infiltrating ductal carcinoma (IDC). 
Table 2: Multivariate analysis of overall survival (OS) and disease-specific survival (DSS) predictors using cox proportional hazard model

\begin{tabular}{|c|c|c|c|c|}
\hline & \multicolumn{2}{|l|}{ OS } & \multicolumn{2}{|c|}{ DSS } \\
\hline & HR (95\% CI) & $P$-Value ${ }^{\text {a }}$ & HR $(95 \%$ CI $)$ & $P$-Value ${ }^{\text {a }}$ \\
\hline \multicolumn{5}{|c|}{ Age at diagnosis (years) } \\
\hline $18-49$ & Reference & - & Reference & - \\
\hline $50-79$ & $1.663(1.615-1.713)$ & $<0.001$ & $1.199(1.160-1.240)$ & $<0.001$ \\
\hline \multicolumn{5}{|l|}{ Race } \\
\hline White & Reference & - & Reference & - \\
\hline Black & $1.276(1.233-1.321)$ & $<0.001$ & $1.293(1.240-1.348)$ & $<0.001$ \\
\hline Others $^{b}$ & $0.756(0.719-0.796)$ & $<0.001$ & $0.778(0.732-0.828)$ & $<0.001$ \\
\hline Unknown & $0.353(0.257-0.486)$ & $<0.001$ & $0.399(0.273-0.582)$ & $<0.001$ \\
\hline \multicolumn{5}{|l|}{ Marital status } \\
\hline Married & Reference & - & Reference & - \\
\hline Not married $^{\mathrm{c}}$ & $1.460(1.422-1.499)$ & $<0.001$ & $1.241(1.201-1.282)$ & $<0.001$ \\
\hline Unknown & $1.119(1.039-1.207)$ & 0.003 & $1.004(0.913-1.105)$ & 0.928 \\
\hline \multicolumn{5}{|l|}{ Histology type } \\
\hline IDC & Reference & - & Reference & - \\
\hline $\mathrm{AAC}$ & $0.834(0.695-1.002)$ & 0.052 & $0.800(0.639-1.001)$ & 0.051 \\
\hline \multicolumn{5}{|l|}{ Grade } \\
\hline I & Reference & - & Reference & - \\
\hline II & $1.252(1.191-1.316)$ & $<0.001$ & $2.257(2.052-2.483)$ & $<0.001$ \\
\hline $\mathrm{III}+\mathrm{UD}^{\mathrm{d}}$ & $1.671(1.588-1.758)$ & $<0.001$ & $3.685(3.351-4.051)$ & $<0.001$ \\
\hline Unknown & $1.161(1.053-1.280)$ & 0.003 & $2.266(1.964-2.615)$ & $<0.001$ \\
\hline \multicolumn{5}{|l|}{ Tumor size (cm) } \\
\hline$\leq 2$ & Reference & - & Reference & - \\
\hline$>2$ and $\leq 5$ & $1.741(1.690-1.794)$ & $<0.001$ & $2.190(2.106-2.278)$ & $<0.001$ \\
\hline$>5$ & $2.994(2.864-3.131)$ & $<0.001$ & $3.891(3.690-4.103)$ & $<0.001$ \\
\hline Unknown & $4.007(3.687-4.355)$ & $<0.001$ & $5.578(5.089-6.114)$ & $<0.001$ \\
\hline \multicolumn{5}{|l|}{ LN status } \\
\hline Negative & Reference & - & Reference & - \\
\hline Positive & $2.014(1.958-2.072)$ & $<0.001$ & $2.775(2.675-2.878)$ & $<0.001$ \\
\hline Unknown & $3.148(2.967-3.339)$ & $<0.001$ & $3.198(2.940-3.478)$ & $<0.001$ \\
\hline \multicolumn{5}{|l|}{ ER status } \\
\hline Negative & Reference & - & Reference & - \\
\hline Positive & $0.743(0.714-0.772)$ & $<0.001$ & $0.683(0.652-0.716)$ & $<0.001$ \\
\hline \multicolumn{5}{|l|}{ PR status } \\
\hline Negative & Reference & - & Reference & - \\
\hline Positive & $0.771(0.743-0.801)$ & $<0.001$ & $0.641(0.611-0.672)$ & $<0.001$ \\
\hline \multicolumn{5}{|l|}{ Surgery type } \\
\hline Mastectomy & Reference & - & Reference & - \\
\hline $\mathrm{BCS}$ & $0.832(0.807-0.857)$ & $<0.001$ & $0.794(0.765-0.824)$ & $<0.001$ \\
\hline Unknown & $1.088(0.828-1.428)$ & 0.546 & $1.517(1.138-2.022)$ & 0.005 \\
\hline
\end{tabular}


Radiation

No

Yes

Reference

$-$

0.779 (0.757-0.802)

$<0.001$

Reference

Unknown

0.942 (0.877-1.011)

0.096

$0.872(0.842-0.903)$

$<0.001$

$\mathrm{AAC}=$ apocrine adenocarcinoma, $\mathrm{IDC}=$ infiltrating ductal carcinoma, $\mathrm{ER}=$ estrogen receptor, $\mathrm{PR}=$ progesterone receptor, HER2 = human epidermal growth factor receptor 2, LN = lymph node, $\mathrm{NOS}=$ no other specific, $\mathrm{BCS}=$ breast conserving surgery, $\mathrm{HR}=$ hazard ratio, $\mathrm{CI}=$ confidence interval, DSS $=$ disease-specific survival, OS = overall survival.

${ }^{a} P$-value was determined by multivariate Cox proportional hazard regression model.

' Including American Indian/Alaskan native, and Asian/Pacific Islander, and others-unspecified.

'Including divorced, separated, single (never married), unmarried, domestic partner and widowed.

${ }^{\mathrm{d}}$ Including grade 3 and undifferentiated.

(AAC vs. IDC, grade II tumors in OS analysis, $\mathrm{HR}=1.391$, $95 \% \mathrm{CI}=1.034-1.871$; grade I tumors in DSS analysis, $\mathrm{HR}=4.001,95 \% \mathrm{CI}=1.286-12.450)$. Moreover, AAC patients also showed worse prognosis in subgroup of smaller tumors and positive LN (AAC vs. IDC, tumor size $\leq 2 \mathrm{~cm}, \mathrm{HR}=1.449,95 \% \mathrm{CI}=1.081-1.942$ (OS); $\mathrm{HR}=1.838,95 \% \mathrm{CI}=1.250-2.703(\mathrm{DSS}) ;$ positive $\mathrm{LN}$, $\mathrm{HR}=1.378,95 \% \mathrm{CI}=1.099-1.726(\mathrm{OS}) ; \mathrm{HR}=1.448$, $95 \% \mathrm{CI}=1.128-1.859(\mathrm{DSS}))$. For different treatments, AAC patients were presented with worse prognosis in mastectomy and radiation recipients (AAC vs. IDC, mastectomy, $\mathrm{HR}=1.319,95 \% \mathrm{CI}=1.049-1.657(\mathrm{OS})$; $\mathrm{HR}=1.340,95 \% \mathrm{CI}=1.023-1.755(\mathrm{DSS})$; radiation recipients, $\mathrm{HR}=1.444,95 \% \mathrm{CI}=1.121-1.861$ (OS); $\mathrm{HR}=1.534,95 \% \mathrm{CI}=1.137-2.070$ (DSS), respectively).

A forest plot of HRs that was used to illustrate the exploratory subgroup analyses suggested that in some subgroups, an AAC subtype was not a significant positive indicator of DSS or OS any longer (Figure 3). Specifically, HRs in different AJCC stage, ER status, PR

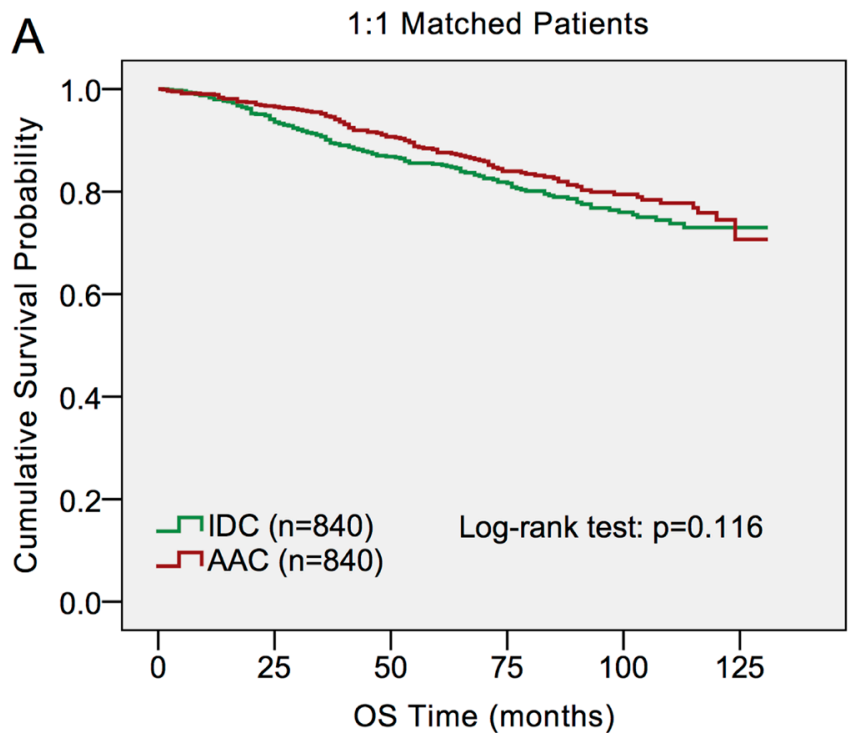

status and HER2 status subgroups were not significantly different between AAC and IDC in subgroup analysis of OS. Similarly, HRs in different AJCC stage, PR status and HER2 status subgroups showed no significant difference between AAC and IDC for DSS. These results suggested that AJCC stage, ER status, PR status and HER2 status may be principal confounders for AAC prognosis.

\section{Stratification analysis with molecular subtype}

Aiming to investigate the role of molecular subtype on breast cancer outcomes between AAC and IDC patients, a multivariate analysis stratified by molecular subtype was performed. As shown in Table 5, HR+/HER2AAC patients showed poorer DSS than HR+/HER2- IDC patients $(\mathrm{HR}=4.110,95 \% \mathrm{CI}: 1.026-16.465, p=0.046)$. Triple-negative (TN)-AAC patients presented better DSS as well as OS than TN-IDC patients $(\mathrm{HR}=0.203$, 95\% CI: $0.051-0.812, p=0.024$ (DSS); HR $=0.254$, 95\% CI: $0.082-0.787, p=0.018$ (OS), respectively).

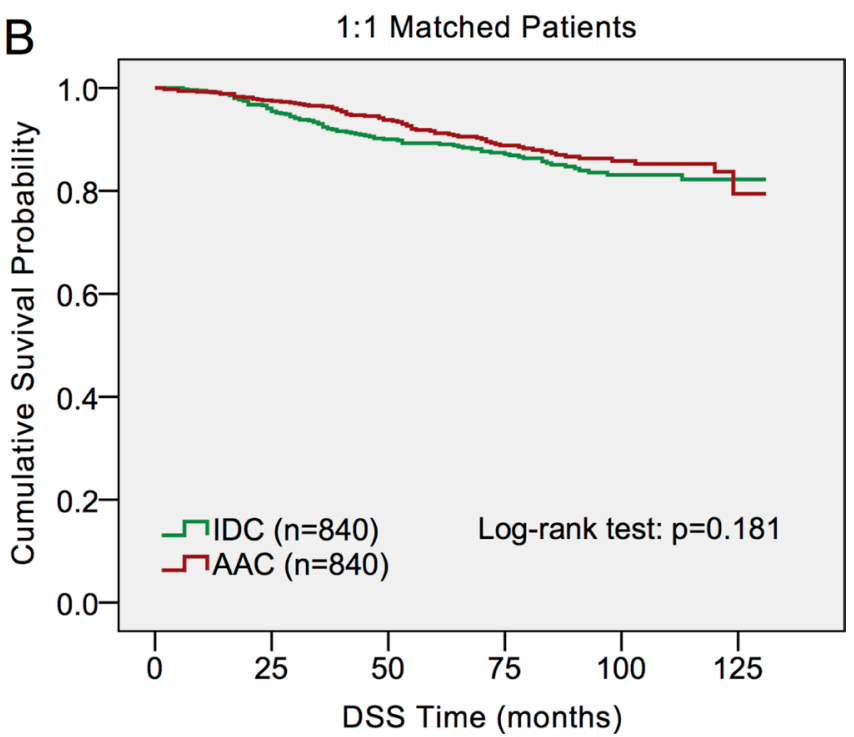

Figure 2: Log-rank test of 1:1 matched groups to compare invasive apocrineadenocarcinoma (AAC) to infiltrating ductal carcinoma (IDC). 
Table 3: Characteristics of patients by histology subtype in 1:1 matched, AAC versus IDC

\begin{tabular}{|c|c|c|c|c|}
\hline & AAC, $n=840$ & IDC, $n=840$ & Total, $n=1,680$ & \multirow{2}{*}{$P$-Value ${ }^{\text {a }}$} \\
\hline & $(\%)$ & $(\%)$ & $(\%)$ & \\
\hline Median follow-up (months) (IQR) & $61(31.25-94)$ & $60(29-92)$ & $61(30-93)$ & \\
\hline \multicolumn{5}{|l|}{ Age at diagnosis (years) } \\
\hline $18-49$ & $159(18.9)$ & $151(18.0)$ & $310(18.4)$ & 0.615 \\
\hline $50-79$ & $681(81.1)$ & $689(82.0)$ & $1,370(81.6)$ & \\
\hline \multicolumn{5}{|l|}{ Race } \\
\hline White & $635(75.6)$ & $613(73.0)$ & $1,248(74.3)$ & 0.199 \\
\hline Black & $99(11.8)$ & $124(14.8)$ & $223(13.3)$ & \\
\hline Others $^{\mathrm{b}}$ & $104(12.4)$ & $98(11.7)$ & $202(12.0)$ & \\
\hline Unknown & $2(0.2)$ & $5(0.6)$ & $7(0.4)$ & \\
\hline \multicolumn{5}{|l|}{ Marital status } \\
\hline Married & $496(59.0)$ & $504(60.0)$ & $1,000(59.5)$ & 0.368 \\
\hline Not married ${ }^{c}$ & $321(38.2)$ & $304(36.2)$ & $625(37.2)$ & \\
\hline Unknown & $23(2.70)$ & $32(3.80)$ & $55(3.3)$ & \\
\hline \multicolumn{5}{|l|}{ Laterality } \\
\hline Left & $434(51.7)$ & $410(48.8)$ & $844(50.2)$ & 0.314 \\
\hline Right & $406(48.3)$ & $429(51.1)$ & 835 (49.7) & \\
\hline Only one side, NOS & $0(0.0)$ & $1(0.1)$ & $1(0.1)$ & \\
\hline \multicolumn{5}{|l|}{ Grade } \\
\hline I & $59(7.0)$ & $81(9.6)$ & $140(8.3)$ & $<0.001$ \\
\hline II & $390(46.4)$ & $313(37.3)$ & $703(41.8)$ & \\
\hline $\mathrm{III}+\mathrm{UD}^{\mathrm{d}}$ & $363(43.2)$ & $433(51.5)$ & $796(47.4)$ & \\
\hline Unknown & $28(3.3)$ & $13(1.5)$ & $41(2.4)$ & \\
\hline \multicolumn{5}{|l|}{ Tumor size $(\mathrm{cm})$} \\
\hline$\leq 2$ & $483(57.5)$ & $511(60.8)$ & $994(59.2)$ & 0.034 \\
\hline$>2$ and $\leq 5$ & $285(33.9)$ & $270(32.1)$ & $555(33.0)$ & \\
\hline$>5$ & $62(7.4)$ & $58(6.9)$ & $120(7.1)$ & \\
\hline Unknown & $10(1.2)$ & $1(0.1)$ & $11(0.7)$ & \\
\hline \multicolumn{5}{|l|}{ LN status } \\
\hline Negative & 497 (59.2) & $496(59.0)$ & $993(59.1)$ & 0.148 \\
\hline Positive & $314(37.4)$ & $299(35.6)$ & $613(36.5)$ & \\
\hline Unknown & $29(3.5)$ & $45(5.4)$ & $74(4.4)$ & \\
\hline \multicolumn{5}{|l|}{ AJCC stage } \\
\hline I & $380(45.2)$ & $406(48.3)$ & $786(46.8)$ & 0.363 \\
\hline II & $312(37.1)$ & $303(36.1)$ & $615(36.6)$ & \\
\hline III & 148 (17.6) & $131(15.6)$ & 279 (16.6) & \\
\hline \multicolumn{5}{|l|}{ ER status } \\
\hline Negative & $589(70.1)$ & $578(68.8)$ & $1,167(69.5)$ & 0.560 \\
\hline Positive & 251 (29.9) & $262(31.2)$ & $513(30.5)$ & \\
\hline \multicolumn{5}{|l|}{ PR status } \\
\hline Negative & $645(76.8)$ & $635(75.6)$ & $1,280(76.2)$ & 0.567 \\
\hline Positive & $195(23.2)$ & $205(24.4)$ & $400(23.8)$ & \\
\hline
\end{tabular}




\begin{tabular}{lcccc} 
HER2 status & & & & \\
Negative & $187(22.3)$ & $175(20.8)$ & $362(21.5)$ & 0.838 \\
Positive & $64(7.6)$ & $60(7.1)$ & $124(7.4)$ & \\
Borderline & $7(0.8)$ & $6(0.7)$ & $13(0.8)$ & \\
Unknown & $582(69.3)$ & $599(71.3)$ & $1,181(70.3)$ & \\
Surgery type & & & & \\
Mastectomy & $377(44.9)$ & $354(42.1)$ & $731(43.5)$ & 0.527 \\
BCS & $462(55.0)$ & $485(57.7)$ & $947(56.4)$ & \\
Unknown & $1(0.1)$ & $1(0.1)$ & $2(0.1)$ & \\
Radiation & & & & \\
No & $365(43.5)$ & $350(41.7)$ & $715(42.6)$ & 0.717 \\
Yes & $440(52.4)$ & $451(53.7)$ & $891(53.0)$ & \\
Unknown & $35(4.2)$ & $39(4.6)$ & $74(4.4)$ & \\
\hline
\end{tabular}

$\mathrm{AJCC}=$ American Joint Committee on Cancer, BCS = breast conserving surgery, ER = estrogen receptor, PR = progesterone receptor, HER2 $=$ human epidermal growth factor receptor $2, \mathrm{IDC}=$ infiltrating ductal carcinoma, AAC $=$ apocrine adenocarcinoma, $\mathrm{IQR}=$ interquartile range, $\mathrm{LN}=$ lymph node, $\mathrm{NOS}=$ no other specific, $\mathrm{UD}=$ undifferentiated.

${ }^{a} P$-valuedetermined by Chi-square test comparing the matched AAC and IDC groups.

bIncluding American Indian/Alaskan native, and Asian/Pacific Islander, and others-unspecified.

'Including divorced, separated, single (never married), unmarried, domestic partner and widowed.

Including grade 3 and undifferentiated.
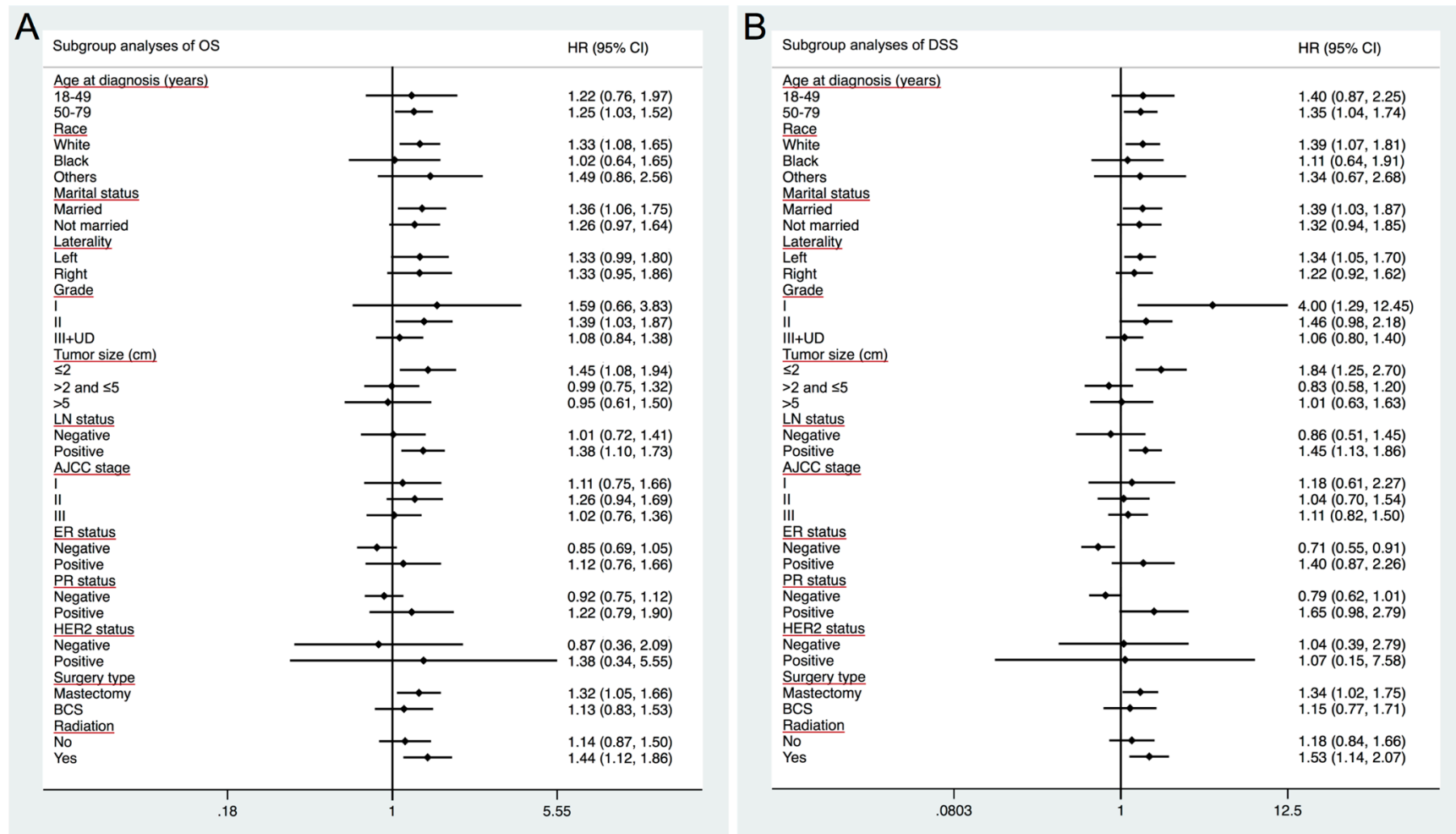

Figure 3: Forest plot of hazard ratios (HRs) for invasive apocrine adenocarcinoma (AAC) versus infiltrating ductal carcinoma (IDC) in the subgroup analysis. The diamond on the $\mathrm{X}$-axis indicates the HR and the $95 \%$ confident interval (CI) of each subgroup. 
Table 4: Comparison of overall survival (OS) and disease-specific survival (DSS) between AAC vs IDC after subgroup analyses by univariate cox proportional hazard model

\begin{tabular}{|c|c|c|c|c|}
\hline & \multicolumn{2}{|c|}{ OS } & \multicolumn{2}{|l|}{ DSS } \\
\hline & HR (95\% CI) & $P$-Value ${ }^{\text {a }}$ & HR $(95 \%$ CI $)$ & $P$-Value ${ }^{\text {a }}$ \\
\hline \multicolumn{5}{|c|}{ Age at diagnosis (years) } \\
\hline $18-49$ & $1.222(0.759-1.967)$ & 0.409 & $1.397(0.868-2.249)$ & 0.169 \\
\hline $50-79$ & $1.252(1.028-1.525)$ & 0.026 & $1.347(1.045-1.736)$ & 0.022 \\
\hline \multicolumn{5}{|l|}{ Race } \\
\hline White & $1.332(1.078-1.646)$ & 0.008 & $1.392(1.071-1.810)$ & 0.014 \\
\hline Black & $1.024(0.636-1.649)$ & 0.923 & $1.106(0.641-1.907)$ & 0.717 \\
\hline Others $^{\mathrm{b}}$ & $1.485(0.860-2.563)$ & 0.156 & $1.336(0.666-2.678)$ & 0.415 \\
\hline \multicolumn{5}{|l|}{ Marital status } \\
\hline Married & $1.363(1.060-1.752)$ & 0.016 & $1.388(1.028-1.872)$ & 0.032 \\
\hline Not married & $1.261(0.968-1.644)$ & 0.086 & $1.320(0.943-1.849)$ & 0.106 \\
\hline \multicolumn{5}{|l|}{ Laterality } \\
\hline Left & $1.332(0.987-1.798)$ & 0.061 & $1.337(1.052-1.700)$ & 0.018 \\
\hline Right & $1.328(0.948-1.860)$ & 0.099 & $1.223(0.924-1.619)$ & 0.160 \\
\hline \multicolumn{5}{|l|}{ Grade } \\
\hline $\mathrm{I}$ & $1.592(0.662-3.828)$ & 0.299 & $4.001(1.286-12.450)$ & 0.017 \\
\hline II & $1.391(1.034-1.871)$ & 0.029 & $1.462(0.978-2.183)$ & 0.064 \\
\hline $\mathrm{III}+\mathrm{UD}^{\mathrm{c}}$ & $1.079(0.844-1.380)$ & 0.543 & $1.056(0.795-1.402)$ & 0.706 \\
\hline \multicolumn{5}{|l|}{ Tumor size $(\mathrm{cm})$} \\
\hline$\leq 2$ & $1.449(1.081-1.942)$ & 0.013 & $1.838(1.250-2.703)$ & 0.002 \\
\hline $2-5$ & $0.994(0.747-1.324)$ & 0.970 & $0.834(0.579-1.201)$ & 0.330 \\
\hline$>5$ & $0.955(0.609-1.500)$ & 0.842 & $1.012(0.628-1.630)$ & 0.961 \\
\hline \multicolumn{5}{|l|}{ LN status } \\
\hline Negative & $1.010(0.721-1.414)$ & 0.953 & $0.857(0.507-1.448)$ & 0.564 \\
\hline Positive & $1.378(1.099-1.726)$ & 0.005 & $1.448(1.128-1.859)$ & 0.004 \\
\hline \multicolumn{5}{|l|}{ AJCC stage } \\
\hline I & $1.114(0.746-1.664)$ & 0.597 & $1.180(0.613-2.271)$ & 0.620 \\
\hline II & $1.263(0.942-1.692)$ & 0.119 & $1.042(0.704-1.543)$ & 0.837 \\
\hline III & $1.019(0.765-1.357)$ & 0.900 & $1.114(0.825-1.504)$ & 0.480 \\
\hline \multicolumn{5}{|l|}{ ER status } \\
\hline Negative & $0.852(0.693-1.048)$ & 0.129 & $0.709(0.550-0.914)$ & 0.008 \\
\hline Positive & $1.121(0.757-1.659)$ & 0.568 & $1.402(0.871-2.256)$ & 0.164 \\
\hline \multicolumn{5}{|l|}{ PR status } \\
\hline Negative & $0.918(0.751-1.122)$ & 0.405 & $0.792(0.618-1.014)$ & 0.064 \\
\hline Positive & $1.223(0.789-1.896)$ & 0.368 & $1.649(0.976-2.787)$ & 0.061 \\
\hline \multicolumn{5}{|l|}{ HER2 status } \\
\hline Negative & $0.867(0.361-2.085)$ & 0.750 & $1.044(0.391-2.785)$ & 0.931 \\
\hline Positive & $1.384(0.345-5.550)$ & 0.647 & $1.065(0.149-7.584)$ & 0.950 \\
\hline \multicolumn{5}{|l|}{ Surgery type } \\
\hline Mastectomy & $1.319(1.049-1.657)$ & 0.018 & $1.340(1.023-1.755)$ & 0.034 \\
\hline BCS & $1.129(0.834-1.529)$ & 0.431 & $1.147(0.768-1.712)$ & 0.504 \\
\hline
\end{tabular}


Radiation

No

$1.142(0.872-1.496)$

0.334

$1.179(0.838-1.660)$

0.345

Yes

1.444 (1.121-1.861)

0.005

$1.534(1.137-2.070)$

0.005

AJCC $=$ American Joint Committee on Cancer, AAC $=$ apocrine adenocarcinoma, IDC $=$ infiltrating ductal carcinoma, $\mathrm{ER}=$ estrogen receptor, $\mathrm{PR}=$ progesterone receptor, HER2 = human epidermal growth factor receptor $2, \mathrm{LN}=$ lymph node, $\mathrm{BCS}=$ breast conserving surgery, $\mathrm{HR}=$ hazard ratio, $\mathrm{CI}=$ confidence interval, $\mathrm{DSS}=$ disease-specific survival, $\mathrm{OS}=$ overall survival.

${ }^{a} P$-value was determined by univariate Cox proportional hazard regression model.

${ }^{b}$ Including American Indian/Alaskan native, and Asian/Pacific Islander, and others-unspecified.

'Including grade 3 and undifferentiated.

\section{DISCUSSION}

Apocrine carcinoma is a very rare and unique neoplasm of the breast, which is a morphologically distinct type from IDC. As it accounts for very small amount of all breast cancer, a large population is needed to obtain a sufficient number of patients with the relatively rare tumors. Therefore, we retrospectively investigated the clinicopathological and prognostic features of AAC in SEER database. Our findings indicated that AAC had unique clinicopathological characteristics and it tended to be a more aggressive type than IDC. Consistently, OS and DSS were both worse in ACC patients than in IDC patients with Kaplan-Meier analysis. Nevertheless, AAC patients presented similar survival outcomes in both OS and DSS to IDC after matching baseline characteristics. AAC histology was found not to be an independent prognostic factor after multivariate analysis in Cox proportional hazard model either. Further subgroup analysis indicated that AJCC stage, ER status, PR status and HER2 status may be principal confounders for AAC prognosis.

To our best knowledge, this study contained the largest number of patients compared to other published studies. We summarized both demographic and clinicopathological characteristics of AAC and found that this unique histological type was associated with an older age, lower proportion of white race, a larger tumor size, a higher grade, more positive LNs, an aggressive stage, lower ER and PR proportions, and higher HER2 amplification rates than that of IDC. As for the treatment strategies, AAC patients were less likely to be treated with breast-conserving surgery and adjuvant radiotherapy. These observations were partially in concordance with previous studies. Matsuo et al. reported that in patients with AAC, older age and postmenopausal status were observed more frequently than in those with IDC [9]. The study of Tanaka et al. and Dreyer et al. also confirmed that the patients with AAC were older $[10,11]$. However, no significant difference with regard to menopausal status was observed between the two groups [10]. According to some literatures, AAC showed some non-aggressive behavior: axillary lymph node metastasis varies from $<1 \%$ to $4 \%$ [2]. Tanaka et al. also reported the proportion of AAC with LN metastasis and lymphatic invasion were significantly lower in the AAC patients than in the IDC patients [10]. In contrast, Dreyer et al. showed 7 out of 14 apocrine breast carcinomas with positive lymph node status [11] and Choi et al. reported the rage of lymph node metastasis was highest in the molecular apocrine type, although not statistically significant [21]. The inconsistence might be due to the heterogeneity of cohort since the study only involved very small number of patients.

Apocrine differentiation is generally inversely correlated with the expression of ER and PR but shows strong expression of the AR and HER2 or EGFR [3, 14, 22]. Moreover, expression of GCDFP-15 and CK20 is reported $[23,24]$. In our present study, lower ER and PR proportions of AAC were reported. Consistently, several studies showed parallel results that the percentage of ER and PR receptor negativity was higher in the AAC group than in the IDC group $[10,17]$. In addition, more positive HER2 status was shown in AAC patients than in IDC patients ( $7.6 \%$ vs. $6.8 \%$, respectively; $p<0.001)$, which was consistent with others' results [25]. Besides, some reported AAC was found very frequently in the triplenegative breast cancer (TNBC) group [17].

Most of previous studies revealed that AAC had a similar or more favorable prognosis when compared with IDC $[8,10,15-18]$. For example, Tanaka et al. reported $12 \%$ patients with AAC and $15 \%$ patients with IDC had experienced recurrences, while $5 \%$ patients with $\mathrm{AAC}$ and $8 \%$ patients with IDC died of recurrent breast cancer after a median follow-up period of 49 months. No significant differences in the relapse-free survival $(p=0.83)$ and overall survival $(p=0.75)$ rates were observed between the two groups [10]. Furthermore, Takeuchi et al. demonstrated the clinicopathological factors influencing 12 -year survival rate were lymph node metastasis, lymphatic involvement and vascular involvement [17]. Although AAC and IDC had different clinicopathological characteristics, there was no difference in survival rates at 10 years after operation between AAC and non-AAC patients [17]. In the present study, we observed that OS and DSS were both worse in ACC patients than in IDC patients. Nevertheless, AAC patients presented similar survival outcomes in both OS and DSS to IDC when each AAC was matched with one IDC according to the most important prognostic parameters: age at diagnosis, 
Table 5: Comparison of disease-specific survival (DSS) and overall survival (OS) between AAC vs IDC after breast subtype analyses by univariate cox proportional hazard model

\begin{tabular}{|c|c|c|c|c|c|c|}
\hline \multirow[b]{2}{*}{ Subtype } & \multicolumn{3}{|c|}{ DSS } & \multicolumn{3}{|c|}{ OS } \\
\hline & $\begin{array}{l}\text { Events No./ } \\
\text { Sum No. }\end{array}$ & HR (95\% CI) & $P$-Value ${ }^{a}$ & $\begin{array}{l}\text { Events No./ } \\
\text { Sum No. }\end{array}$ & $\operatorname{HR}(95 \% \mathrm{CI})$ & $P$-Value ${ }^{\text {a }}$ \\
\hline \multicolumn{7}{|l|}{ HR+/HER2- } \\
\hline $\mathrm{AAC}$ & $2 / 51$ & $4.110(1.026-16.465)$ & 0.046 & $2 / 51$ & $2.174(0.543-8.702)$ & 0.272 \\
\hline IDC & $724 / 72,454$ & Reference & & $1,363 / 72,454$ & Reference & \\
\hline \multicolumn{7}{|l|}{ HR+/HER2+ } \\
\hline $\mathrm{AAC}$ & $0 / 22$ & - & - & $0 / 22$ & - & - \\
\hline IDC & $129 / 12,268$ & Reference & & $231 / 12,268$ & Reference & \\
\hline \multicolumn{7}{|l|}{ HR-/HER2+ } \\
\hline $\mathrm{AAC}$ & $1 / 42$ & $0.907(0.127-6.479)$ & 0.923 & $2 / 42$ & $1.382(0.343-5.562)$ & 0.649 \\
\hline IDC & $163 / 5,472$ & Reference & & $213 / 5,472$ & Reference & \\
\hline \multicolumn{7}{|c|}{ Triple negative } \\
\hline $\mathrm{AAC}$ & $2 / 136$ & $0.203(0.051-0.812)$ & 0.024 & $3 / 136$ & $0.254(0.082-0.787)$ & 0.018 \\
\hline IDC & $860 / 13,837$ & Reference & & $1,039 / 13,837$ & Reference & \\
\hline
\end{tabular}

pathological grade, tumor size, regional nodal status, ER status, PR status, HER2 status, treatment strategies and so on. Additionally, AAC histology was found not to be an independent prognostic factor after multivariate analysis in Cox proportional hazard model either. Interestingly, Nagao et al. reported AAC responded poorly to neoadjuvant chemotherapy (NAC). Despite their poor response to NAC treatment, patients with AAC was reported to have a good prognosis [15]. In accordance, Aoyagi et al. and Japaze et al. also reported that a significantly better outcome was observed in patients with AAC $[12,13]$.

In order to investigate the role of molecular subtype on prognoses between AAC and IDC patients, a multivariate analysis stratified by molecular subtype was performed as well. Interestingly, TN-AAC patients presented better DSS and OS outcomes than TN-IDC patients $(p=0.024)$. These results were in accordance with the findings of Iwase et al. and Choi et al. that AAC had better prognosis than non-AAC among TN breast cancers $[21,26]$. Thus Iwase et al. suggested AAC should be regarded as different from the more common basal-like breast cancer [26]. Other studies suggested no significant differences if AAC compared with ductal carcinomas [20]. Notably, some of the histological subgroups contained insufficient numbers in order to draw firm conclusions [11].

The results of this study have several therapeutic implications. Since histological type was not an independent prognostic factor in the multivariate analysis, treatment guidelines do not need to be specified made based on this rare entity. Furthermore, since the subgroup analyses suggested that AJCC stage, ER status, PR status and HER2 status are the principal confounders for AAC prognosis; doctors should take more account of these prognostic indicators other than histological types.

Considering the similar prognostic outcomes of AAC to IDC, the existence of AAC of the breast as a distinct clinicopathological entity is debatable [27]. One strong reason for a designation of $\mathrm{AAC}$ is the identification of a subgroup of breast carcinomas that appear to have a unique response to androgen stimuli [28]. As an ER-positive breast cancer may benefit from estrogen deprivation treatment, some studies demonstrates that an AR-expressing AAC may respond to androgen deprivation [27]. It highlights the importance of further research elucidating the AR pathway in AAC, for which androgen represents the known steroid hormone stimulating tumor growth [29].

Inevitably, our study has several limitations. It has been widely accepted that AAC shows a characteristic steroid receptor profile: ER negative, PR negative, AR positive, and HER-2 or EGFR positive [25]. However, the status of HER-2 expression was not available until 2010 in SEER database. The status of AR and EGFR were not essential criteria for the diagnosis of AAC, therefore their expression were not recorded routinely in SEER database. Additionally, information regarding adjuvant chemotherapy and adjuvant endocrine therapy is absent from SEER database, which are both important prognostic factors for breast cancer. Other clinical parameters, like body mass index (BMI), age at first birth, family history 
of breast cancer as well as Eastern Cooperative Oncology Group (ECOG) scores need further investigation when these data are available in the future.

Collectively, we investigated a large population of patients with AAC and indicated that this rare histological type had unique clinicopathological characteristics and it tended to be a more aggressive type than that were observed in IDC patients. However, the worse prognosis was attenuated after adjusting for demographic and clinicopathological factors in the multivariate analysis. These results not only improve our understanding of the clinicopathological and prognostic features of this rare entity but also provide more convincing therapeutic guidelines for $\mathrm{AAC}$ of breast cancer patients.

\section{MATERIALS AND METHODS}

\section{Ethics statement}

We used National Cancer Institute's SEER data research files released in Nov 2015, which includes cancer registries covering $28 \%$ of the U.S. population. The data released by the SEER database do not require informed patient consent since cancer is a reportable disease in the United States. We obtained the permission to access the SEER database with the ID number 10444-Nov2015 via Internet access method. Our study was approved by the Ethical Committee of Shandong University.

\section{Patients selection}

In order to identify eligible patients, we use the inclusion criteria as follows: female aged between 18 and 79 , unilateral breast cancer, breast cancer (ICD-O-3 site code C50) as the first and only cancer diagnosis, diagnosis not obtained from a death certificate or autopsy, pathologic confirmation of infiltrating ductal carcinoma (IDC), not otherwise specified (ICD-O-3 8500/3) and apocrine adenocarcinoma (AAC) (ICD-O-3 8401/3)with invasion (behavior codeICD-O-3 malignant), known ER and PR statuses, American Joint Committee on Cancer (AJCC) stages I-III, and diagnosis from January 1, 2003 to December 31, 2013. We use the SEER*stat version 8.3.2 to generate a case-listing file. Finally, a total of 260,596 patients were included in our study. Of these patients, 840 were diagnosed with AAC and 259,756 with IDC.

Demographic characteristics included age at diagnosis, race, and marital status. We treated age at diagnosis as a binary variable classified into two groups: 18-49 years and 50-79 years. Tumor statistics included laterality, histological grade, tumor size, regional lymph node (LN) status, AJCC stage, ER status, PR status, and HER2 status. The evaluations of ER, PR as well as HER2 status were based on the guidelines from the American Society of Clinical Oncology (ASCO) and College of American Pathologists (CAP). Among those variables, known tumor size was treated as a categorical variable classified into the following groups: $\leq 2 \mathrm{~cm},>2 \mathrm{~cm}$ and $\leq 5 \mathrm{~cm}$, or $>5 \mathrm{~cm}$. Due to the limitation of the SEER data files, HER2 status was only available until 2010 for both subtypes.

\section{Statistical analysis}

Clinicopathological characteristics were compared between groups using Pearson's Chi-square test. Survival curves were generated using the Kaplan-Meier method, and differences between curves were analyzed with logrank test. Multivariate Cox proportional hazard model was applied to estimate the association of covariates with overall survival (OS) and disease-specific survival (DSS). Subgroup analyses using univariate Cox proportional hazard model estimated the HRs of AAC versus IDC, and a forest plot was created to better present each prognostic factor's effect on OS and DSS. Hazard ratios (HRs) and 95\% confidential intervals (CIs) were reported. These above statistical analyses were performed with SPSS version 18.0 (IBM SPSS Statistics, Chicago, IL, US).

To account for differences in baseline characteristics between groups, we matched 1 AAC patient with 1 IDC patient using the following predetermined factors: age at diagnosis, race, marital status, laterality, pathological grade, tumor size, regional LN status, AJCC stage, ER status, PR status, HER2 status, surgery type and radiation type. We utilized psmatch2 code in Stata version 12.0 (StataCorp, College Station, TX, US), which was designed for the propensity score matching method and to test the matching quality for the balance of the match. Two-sided $p$-value $<0.05$ was considered as statistically significant.

\section{ACKNOWLEDGMENTS AND FUNDING}

This study was supported by the National Natural Science Foundation of China (No. 81272903; No. 81672613), Key Research and Development Program of Shandong Province (No. 2016GGE2775) and Special Support Plan for National High Level Talents ("Ten Thousand Talents Program") to Qifeng Yang and National Natural Science Foundation of China (No. 81502285) and the Scientific Research Foundation of Shandong Province for Outstanding Young Scientist Award (No. BS2015YY044) to Ning Zhang.

\section{CONFLICTS OF INTEREST}

The authors declare no conflicts of interests.

\section{Authors' contributions}

N.Z. and Y.Q. designed the study, N.Z., H.Z. and T.C. analyzed the data and wrote the manuscript, and N.Z. and H.Z. draw the figures. All authors reviewed the manuscript. 


\section{REFERENCES}

1. Vranic S, Marchio C, Castellano I, Botta C, Scalzo MS, Bender RP, Payan-Gomez C, di Cantogno LV, Gugliotta P, Tondat F, di Celle PF, Mariani S, Gatalica Z, et al. Immunohistochemical and molecular profiling of histologically defined apocrine carcinomas of the breast. Hum Pathol. 2015; 46:1350-9. doi: 10.1016/j.humpath.2015.05.017.

2. Vranic S, Tawfik O, Palazzo J, Bilalovic N, Eyzaguirre E, Lee LM, Adegboyega P, Hagenkord J, Gatalica Z. EGFR and HER-2/neu expression in invasive apocrine carcinoma of the breast. Mod Pathol. 2010; 23:644-53. doi: 10.1038/ modpathol.2010.50.

3. Vranic S, Schmitt F, Sapino A, Costa JL, Reddy S, Castro M, Gatalica Z. Apocrine carcinoma of the breast: a comprehensive review. Histol Histopathol. 2013; 28:1393-409.

4. O'Malley FP, Bane A. An update on apocrine lesions of the breast. Histopathology. 2008; 52:3-10. doi: 10.1111/j.13652559.2007.02888.x.

5. Celis JE, Cabezon T, Moreira JM, Gromov P, Gromova I, Timmermans-Wielenga V, Iwase T, Akiyama F, Honma N, Rank F. Molecular characterization of apocrine carcinoma of the breast: validation of an apocrine protein signature in a well-defined cohort. Mol Oncol. 2009; 3:220-37. doi: 10.1016/j.molonc.2009.01.005.

6. Celis JE, Gromov P, Cabezon T, Moreira JM, Friis E, Jirstrom K, Llombart-Bosch A, Timmermans-Wielenga V, Rank F, Gromova I. 15-prostaglandin dehydrogenase expression alone or in combination with ACSM1 defines a subgroup of the apocrine molecular subtype of breast carcinoma. Mol Cell Proteomics. 2008; 7:1795-809. doi: 10.1074/mcp.R800011-MCP200.

7. Frable WJ, Kay S. Carcinoma of the breast. Histologic and clinical features of apocrine tumors. Cancer. 1968; 21: 756-63.

8. Mossler JA, Barton TK, Brinkhous AD, McCarty KS, Moylan JA, McCarty KS Jr. Apocrine differentiation in human mammary carcinoma. Cancer. 1980; 46:2463-71.

9. Matsuo K, Fukutomi T, Tsuda H, Kanai Y, Tanaka SA, Nanasawa T. Apocrine Carcinoma of the Breast: Clinicopathological Analysis and Histological Subclassification of 12 Cases. Breast Cancer. 1998; 5:279-84.

10. Tanaka K, Imoto S, Wada N, Sakemura N, Hasebe K. Invasive apocrine carcinoma of the breast: clinicopathologic features of 57 patients. Breast J. 2008; 14:164-8. doi: 10.1111/j.1524-4741.2007.00548.x.

11. Dreyer G, Vandorpe T, Smeets A, Forceville K, Brouwers B, Neven P, Janssens H, Deraedt K, Moerman P, Van Calster B, Christiaens MR, Paridaens R, Wildiers H. Triple negative breast cancer: clinical characteristics in the different histological subtypes. Breast. 2013; 22:761-6. doi: 10.1016/j.breast.2013.01.009.

12. Japaze H, Emina J, Diaz C, Schwam RJ, Gercovich N, Demonty G, Morgenfeld E, Rivarola E, Gil Deza E, Gercovich FG. 'Pure' invasive apocrine carcinoma of the breast: a new clinicopathological entity? Breast. 2005; 14:3-10. doi: 10.1016/j.breast.2004.06.003.

13. Aoyagi H, Ishida T, Yamada I, Kurebayashi J, Kurosumi M, Yokoe T, Ogawa T, Kawai T, Izuo M. [Ten cases of an apocrine carcinoma of the breast and a review of cases in the Japanese literature]. [Article in Japanese]. Gan No Rinsho. 1990; 36:681-90.

14. Ogiya A, Horii R, Osako T, Ito Y, Iwase T, Eishi Y, Akiyama F. Apocrine metaplasia of breast cancer: clinicopathological features and predicting response. Breast Cancer. 2010; 17:290-7. doi: 10.1007/s12282-009-0178-9.

15. Nagao $T$, Kinoshita $T$, Hojo $T$, Tsuda $H$, Tamura $K$, Fujiwara Y. The differences in the histological types of breast cancer and the response to neoadjuvant chemotherapy: the relationship between the outcome and the clinicopathological characteristics. Breast. 2012; 21:289-95. doi: 10.1016/j. breast.2011.12.011.

16. Abati AD, Kimmel M, Rosen PP. Apocrine mammary carcinoma. A clinicopathologic study of 72 cases. Am J Clin Pathol. 1990; 94:371-7.

17. Takeuchi H, Tsuji K, Ueo H, Kano T, Maehara Y. Clinicopathological feature and long-term prognosis of apocrine carcinoma of the breast in Japanese women. Breast Cancer Res Treat. 2004; 88:49-54. doi: 10.1007/s10549004-9495-z.

18. d'Amore ES, Terrier-Lacombe MJ, Travagli JP, Friedman S, Contesso G. Invasive apocrine carcinoma of the breast: a long term follow-up study of 34 cases. Breast Cancer Res Treat. 1988; 12:37-44.

19. Cha YJ, Jung WH, Koo JS. The clinicopathologic features of molecular apocrine breast cancer. Korean J Pathol. 2012; 46:169-76. doi: 10.4132/KoreanJPathol.2012.46.2.169.

20. Montagna E, Maisonneuve P, Rotmensz N, Cancello G, Iorfida M, Balduzzi A, Galimberti V, Veronesi P, Luini A, Pruneri G, Bottiglieri L, Mastropasqua MG, Goldhirsch A, et al. Heterogeneity of triple-negative breast cancer: histologic subtyping to inform the outcome. Clin Breast Cancer. 2013; 13: 31-9. doi: 10.1016/j.clbc.2012.09.002.

21. Choi J, Jung WH, Koo JS. Clinicopathologic features of molecular subtypes of triple negative breast cancer based on immunohistochemical markers. Histol Histopathol. 2012; 27:1481-93.

22. Dellapasqua S, Maisonneuve P, Viale G, Pruneri G, Mazzarol G, Ghisini R, Mazza M, Iorfida M, Rotmensz N, Veronesi P, Luini A, Goldhirsch A, Colleoni M. Immunohistochemically defined subtypes and outcome of apocrine breast cancer. Clin Breast Cancer. 2013; 13: 95-102. doi: 10.1016/j.clbc.2012.11.004.

23. Honma N, Takubo K, Akiyama F, Sawabe M, Arai T, Younes M, Kasumi F, Sakamoto G. Expression of GCDFP-15 and AR decreases in larger or node-positive apocrine carcinomas of the breast. Histopathology. 2005; 47:195-201. doi: 10.1111/j.1365-2559.2005.02181.x.

24. Lehmann-Che J, Hamy AS, Porcher R, Barritault M, Bouhidel F, Habuellelah H, Leman-Detours S, de 
Roquancourt A, Cahen-Doidy L, Bourstyn E, de Cremoux P, de Bazelaire C, Albiter M, et al. Molecular apocrine breast cancers are aggressive estrogen receptor negative tumors overexpressing either HER2 or GCDFP15. Breast Cancer Res. 2013; 15:R37. doi: 10.1186/bcr3421.

25. Yamazaki M, Nagata Y, Monji S, Shigematsu Y, Baba T, Shimokawa H, Uramoto H, Yamada S, Hanagiri T, Tanaka F. Apocrine carcinoma of the breast. J UOEH. 2011; 33:293-301.

26. Iwase H, Kurebayashi J, Tsuda H, Ohta T, Kurosumi M, Miyamoto K, Yamamoto Y, Iwase T. Clinicopathological analyses of triple negative breast cancer using surveillance data from the Registration Committee of the Japanese Breast Cancer Society. Breast Cancer. 2010; 17:118-24. doi: 10.1007/s12282-009-0113-0.
27. Jongen L, Paridaens R, Floris G, Wildiers H, Neven P. Androgen deprivation by adrenal suppression using lowdose hydrocortisone for the treatment of breast carcinoma with apocrine features: a case report illustrating this new paradigm. Breast Cancer Res Treat. 2016; 155:603-7. doi: 10.1007/s10549-016-3708-0.

28. Mazoujian G. Apocrine carcinoma of the breast. Am J Clin Pathol. 1990; 94:485-6.

29. Onoue S, Katoh T, Chigira H, Matsuo K, Suzuki M, Shibata Y, Maeda M. A Case of Apocrine Carcinoma of the Breast Presenting as Two Cysts. Breast Cancer. 1997; 4: 193-6. 